\title{
ON AN UNCONVENTIONAL VARIATIONAL \\ METHOD FOR SOLVING THE PROBLEM \\ OF LINEAR ELASTICITY WITH NEUMANN OR PERIODIC BOUNDARY CONDITIONS
}

\author{
MICHAL KŘÍŽEK and ZDENĚK MILKA \\ Mathematical Institute of the Czech Academy of Sciences \\ Žitná 25, CS-11567 Prague 1, Czech Republic \\ E-mail:KRIZEK@CSEARN.BITNET
}

\begin{abstract}
A new variational formulation of the linear elasticity problem with Neumann or periodic boundary conditions is presented. This formulation does not require any quotient spaces and is advisable for finite element approximations.
\end{abstract}

1. Introduction. In this paper we give an unconventional variational approach for solving the Neumann problem of linear elasticity on $\Omega \subset \mathbb{R}^{d}, d \in$ $\{1,2,3\}$, where $\Omega$ is a bounded domain with Lipschitz boundary $\partial \Omega$. The solution will be sought in the standard product Sobolev space

$$
\left(H^{1}(\Omega)\right)^{d}=\underbrace{H^{1}(\Omega) \times \ldots \times H^{1}(\Omega)}_{d \text { times }},
$$

with no other restrictions upon the solution. Thus the formulation will be suitable for finite element approximations.

Recall first the classical formulation of the Neumann problem in linear elasticity (the so-called first basic problem of linear elasticity — see [8, p. 95]) for a nonhomogeneous and anisotropic material, in general: Find a displacement $u \in\left(C^{2}(\bar{\Omega})\right)^{d}$ such that, for $i=1, \ldots, d$, we have

$$
-\sum_{j, k, l=1}^{d} \frac{\partial}{\partial x_{j}}\left(c_{i j k l} \varepsilon_{k l}(u)\right)=f_{i} \quad \text { in } \Omega,
$$

1991 Mathematics Subject Classification: 65N30, 73C60.

The paper is in final form and no version of it will be published elsewhere. 


$$
\sum_{j, k, l=1}^{d} n_{j} c_{i j k l} \varepsilon_{k l}(u)=g_{i} \quad \text { on } \partial \Omega,
$$

where $n_{j}$ are components of the outward unit normal to $\partial \Omega$,

$$
\varepsilon_{k l}(v)=\frac{1}{2}\left(\frac{\partial v_{k}}{\partial x_{l}}+\frac{\partial v_{l}}{\partial x_{k}}\right), \quad v=\left(v_{1}, \ldots, v_{d}\right)^{T} \in\left(H^{1}(\Omega)\right)^{d},
$$

$\varepsilon=\left(\varepsilon_{k l}\right)_{k, l=1}^{d}$ is the strain tensor and $c_{i j k l} \in C^{1}(\bar{\Omega})$ are elastic coefficients satisfying

$$
c_{j i k l}=c_{i j k l}=c_{k l i j}, \quad i, j, k, l=1, \ldots, d .
$$

We moreover assume that there exists a constant $C>0$ such that

$$
\sum_{i, j, k, l=1}^{d} c_{i j k l}(x) e_{i j} e_{k l}>C \sum_{i, j=1}^{d} e_{i j}^{2}
$$

for any $x \in \bar{\Omega}$ and any symmetric matrix $\left(e_{i j}\right)_{i, j=1}^{d}, e_{i j} \in \mathbb{R}^{1}$. The body forces $f=$ $\left(f_{1}, \ldots, f_{d}\right)^{T} \in\left(L^{2}(\Omega)\right)^{d}$ and surface forces $g=\left(g_{1}, \ldots, g_{d}\right)^{T} \in\left(L^{2}(\partial \Omega)\right)^{d}$ are supposed to satisfy the following equilibrium condition (for forces and moments):

$$
\int_{\Omega} f^{T} p d x+\int_{\partial \Omega} g^{T} p d s=0 \quad \forall p \in P
$$

where

$$
P=\left\{p \in\left(H^{1}(\Omega)\right)^{d} \mid \varepsilon(p)=0\right\} .
$$

It is known that the space $P$ is finite-dimensional (see e.g. [8, p. 78]),

$$
D \equiv \operatorname{dim} P=\frac{d(d+1)}{2}
$$

$(D=D(d))$. Basis functions $p^{i}=p^{i}\left(x_{1}, \ldots, x_{d}\right), i=1, \ldots, D$, in $P$ can be chosen for instance as follows:

$$
\begin{aligned}
& p^{1}=1 \text { for } d=1, \\
& p^{1}=(1,0)^{T}, p^{2}=(0,1)^{T}, p^{3}=\left(x_{2},-x_{1}\right)^{T} \text { for } d=2, \\
& p^{1}=(1,0,0)^{T}, p^{2}=(0,1,0)^{T}, p^{3}=(0,0,1)^{T}, p^{4}=\left(0, x_{3},-x_{2}\right)^{T}, \\
& p^{5}=\left(-x_{3}, 0, x_{1}\right)^{T}, p^{6}=\left(x_{2},-x_{1}, 0\right)^{T} \text { for } d=3 .
\end{aligned}
$$

We see that if $u$ is a solution of the problem (1.1)-(1.2) then so is $u+p$ for any $p \in P$. Therefore, the standard primal variational formulation of (1.1)-(1.2) is usually given in the quotient space $\left(H^{1}(\Omega)\right)^{d} / P$. However, quotient spaces are not suitable for finite element approximations.

When $d>1$, it is not advisable to look for a finite element solution $u_{h}$ of (1.1)(1.2) which would be fixed at $D$ points. Note that the true solution $u \in\left(H^{1}(\Omega)\right)^{d}$ may have singularities just at some of these points, as $H^{1}(\Omega) \not \subset C(\Omega)$ if $d>1$. Such finite element approximations can then be incorrect (see e.g. [1, p. 13]). 
When we employ a dual variational formulation to (1.1)-(1.2), then there is no trouble with the uniqueness of the solution, but a stress tensor satisfying the equilibrium equations must be known a priori (see [3, p. 50]). This fact often makes the dual approach inapplicable.

Another variational formulation of $(1.1)-(1.2)$ is presented in [8, p. 99]. Here the authors define linear functionals $q_{i}, i=1, \ldots, D$, by

$$
q_{i}(v)=\int_{S} v^{T} p^{i} d s, \quad v \in\left(H^{1}(\Omega)\right)^{d}
$$

where the $p^{i}$ are given by (1.9) and $S \neq \emptyset$ is an arbitrary open part of the boundary $\partial \Omega_{0}$ of a domain $\Omega_{0} \subseteq \Omega$ with Lipschitz boundary. They further prove that the bilinear form

$$
a(v, w)=\int_{\Omega} \sum_{i, j, k, l=1}^{d} c_{i j k l} \varepsilon_{i j}(v) \varepsilon_{k l}(w) d x, \quad v, w \in\left(H^{1}(\Omega)\right)^{d},
$$

associated with the problem (1.1)-(1.2) is $V_{p}$-elliptic for

$$
V_{p}=\left\{v \in\left(H^{1}(\Omega)\right)^{d} \mid q_{i}(v)=0, i=1, \ldots, D\right\} .
$$

However, this space is again unsuitable for finite element approximations due to the constraints $q_{i}(v)=0$.

In Section 2 we will introduce a new variational formulation of (1.1)-(1.2), where the linear functionals $q_{i}$ will appear in a modified bilinear form $\widetilde{a}(\cdot, \cdot)$, but they will not appear in the space of test functions. Section 3 is devoted to finite element approximations. A weak formulation of the Neumann problem with periodic boundary conditions is presented in Section 4 .

2. New variational approach to the Neumann problem in linear elasticity. Throughout the paper the symbols $C, C_{1}, C_{2}$ stand for the so-called generic positive constants which need not be the same at each occurrence. The standard norm in $\left(H^{k}(\Omega)\right)^{m}(k, m$ integers $)$ is denoted by $\|\cdot\|_{k, \Omega}$. Set

$$
V=\left(H^{1}(\Omega)\right)^{d},
$$

and for $c_{i j k l} \in L^{\infty}(\Omega)$ define a symmetric bilinear form

$$
\widetilde{a}(v, w)=a(v, w)+\sum_{i=1}^{D} \gamma_{i} q_{i}(v) q_{i}(w), \quad v, w \in V,
$$

where $\gamma_{i}>0$ are fixed constants, and $D, q_{i}$ and $a(\cdot, \cdot)$ are defined by (1.8), (1.10) and (1.11), respectively. Further, we put

$$
b(v)=\int_{\Omega} f^{T} v d x+\int_{\partial \Omega} g^{T} v d s, \quad v \in V .
$$

We will show that there is a unique solution of the following problem: Find 
$u \in V$ such that

$$
\widetilde{a}(u, v)=b(v) \quad \forall v \in V .
$$

If, moreover, $u \in\left(C^{2}(\bar{\Omega})\right)^{d}$, we shall prove that $u$ solves (1.1)-(1.2). First, we introduce an important lemma.

Lemma 2.1. There exist positive constants $C_{1}, C_{2}$ such that

$$
C_{1}\|v\|_{1, \Omega}^{2} \leq\|\varepsilon(v)\|_{0, \Omega}^{2}+\sum_{i=1}^{D} q_{i}^{2}(v) \leq C_{2}\|v\|_{1, \Omega}^{2} \quad \forall v \in V
$$

where the $q_{i}$ are defined by (1.10).

The proof is based upon Korn's inequality and can be found in [2, p. 309]. For the case $d=3$ see also [8, p. 97].

THEOREM 2.2. There exists a unique solution $u \in V$ of problem (2.3). This solution satisfies the conditions

$$
q_{i}(u)=0, \quad i=1, \ldots, D
$$

where the $q_{i}$ are defined by (1.10).

Proof. From (1.11) and (1.5) we see that there exists a constant $C>0$ such that

$$
a(v, v) \geq C\|\varepsilon(v)\|_{0, \Omega}^{2} \quad \forall v \in V
$$

Moreover, by (1.7),

$$
a(v, p)=0 \quad \forall v \in V \forall p \in P .
$$

(So, in particular, $a(p, p)=0$ for any $p \in P$, i.e., the bilinear form $a(\cdot, \cdot)$ is not $V$-elliptic.) By $(2.1),(2.6)$ and $(2.4)$, we find that $\widetilde{a}(\cdot, \cdot)$ is $V$-elliptic,

$$
\begin{aligned}
\widetilde{a}(v, v) & =a(v, v)+\sum_{i=1}^{D} \gamma_{i} q_{i}^{2}(v) \\
& \geq C\|\varepsilon(v)\|_{0, \Omega}^{2}+\sum_{i=1}^{D} \gamma_{i} q_{i}^{2}(v) \geq C_{1}\|v\|_{1, \Omega}^{2} \quad \forall v \in V .
\end{aligned}
$$

From (1.10), the Cauchy-Schwarz inequality and the trace theorem we see that the $q_{i}$ are continuous,

$$
\left|q_{i}(v)\right| \leq \int_{S}\left|v^{T} p^{i}\right| d s \leq\|v\|_{0, S}\left\|p^{i}\right\|_{0, S} \leq C\|v\|_{1, \Omega}
$$

$$
\forall v \in V, i=1, \ldots, D
$$


Hence, by $(2.1),(1.11),(2.8)$ and $(1.3)$ we deduce that $\widetilde{a}(\cdot, \cdot)$ is also continuous,

$$
\begin{aligned}
|\widetilde{a}(v, w)| & \leq|a(v, w)|+\sum_{i=1}^{D} \gamma_{i}\left|q_{i}(v) \| q_{i}(w)\right| \\
& \leq C_{1}\|\varepsilon(v)\|_{0, \Omega}\|\varepsilon(w)\|_{0, \Omega}+C_{2}\|v\|_{1, \Omega}\|w\|_{1, \Omega} \\
& \leq C\|v\|_{1, \Omega}\|w\|_{1, \Omega} \quad \forall v, w \in V .
\end{aligned}
$$

Since the linear form (2.2) is continuous as well, the existence of a unique $u \in V$ satisfying (2.3) follows from the well-known Lax-Milgram lemma.

Further, we prove (2.5). So, let $u \in V$ be the solution of problem (2.3). Then according to (2.7), (2.1), (2.3), (2.2) and (1.6), we obtain

$$
\sum_{i=1}^{D} \gamma_{i} q_{i}(u) q_{i}(p)=a(u, p)+\sum_{i=1}^{D} \gamma_{i} q_{i}(u) q_{i}(p)=\widetilde{a}(u, p)=b(p)=0
$$

for all $p \in P$. Letting

$$
\alpha_{i}=\gamma_{i} q_{i}(u)
$$

we shall prove that

$$
\sum_{i=1}^{D} \alpha_{i} q_{i}(p)=0 \forall p \in P \quad \Rightarrow \quad \alpha_{i}=0, i=1, \ldots, D .
$$

Let us take, in particular,

$$
p=\sum_{i=1}^{D} \alpha_{i} p^{i}
$$

where the $p_{i}$ are defined by (1.9). Then by (2.9), (2.10), (1.10) and (2.12) we get

$$
0=\sum_{i=1}^{D} \alpha_{i} q_{i}(p)=\sum_{i=1}^{D} \alpha_{i} \int_{S} p^{T} p^{i} d s=\int_{S} p^{T} p d s,
$$

which yields

$$
p=0 \quad \text { on } S .
$$

If $d=1$ then clearly $\alpha_{1}=0$. Consider the case $d=2$. Since $S \neq \emptyset$ is an open part of the boundary $\partial \Omega_{0}$, there exist at least two different points $\left(x_{1}, x_{2}\right)^{T}$ and $\left(y_{1}, y_{2}\right)^{T}$ lying on $S$. So by $(2.12),(1.9)$ and $(2.13)$ we have

$$
\left(\begin{array}{c}
\alpha_{1}+\alpha_{3} x_{2} \\
\alpha_{2}-\alpha_{3} x_{1}
\end{array}\right)=0, \quad\left(\begin{array}{c}
\alpha_{1}+\alpha_{3} y_{2} \\
\alpha_{2}-\alpha_{3} y_{1}
\end{array}\right)=0
$$

which yields $\alpha_{3}=0$ and thus also $\alpha_{1}=\alpha_{2}=0$. For $d=3$ we also have $\alpha_{1}=$ $\ldots=\alpha_{6}=0$, which is proved in [7, p. 91]. Hence (2.11) holds, and by $(2.10)$ we find that $q_{i}(u)=0$ for every $i=1, \ldots, D$.

R e mark 2.3. From (2.9) we see that the equilibrium condition (1.6) in fact implies (2.5). The converse is also true. Namely, by (2.1) and (2.5) we find that 
$\widetilde{a}(u, v)=a(u, v)$ for any $v \in V$, and thus the variational solution $u \in V$ satisfies, by $(2.3)$, the relation

$$
a(u, v)=b(v) \quad \forall v \in V .
$$

From here, (2.7) and (2.2) we get the equilibrium condition (1.6):

$$
0=a(u, p)=b(p)=\int_{\Omega} f^{T} p d x+\int_{\partial \Omega} g^{T} p d s \quad \forall p \in P .
$$

Next we prove two theorems characterizing a connection between the classical and variational Neumann problem.

THEOREM 2.4. Let the coefficients $c_{i j k l}$ occurring in (1.11) belong to $C^{1}(\bar{\Omega})$ and let the variational solution $u$ of problem $(2.3)$ belong to $\left(C^{2}(\bar{\Omega})\right)^{d}$. Then $u$ is also the classical solution of problem (1.1)-(1.2).

Pro of. Denote by $C_{0}^{\infty}(\Omega)$ the space of infinitely differentiable functions with a compact support in $\Omega$. Then by $(2.14)$, the symmetry of $a(\cdot, \cdot),(1.4)$ and by Green's formula

$$
\begin{aligned}
& 0=a(u, v)-b(v)=a(v, u)-b(v) \\
&= \int_{\Omega}\left(\frac{1}{2} \sum_{i, j, k, l} c_{i j k l} \varepsilon_{k l}(u)\left(\frac{\partial v_{i}}{\partial x_{j}}+\frac{\partial v_{j}}{\partial x_{i}}\right)-f^{T} v\right) d x \\
&=-\int_{\Omega}\left(\frac{1}{2} \sum_{i, j, k, l}\left(\frac{\partial}{\partial x_{j}}\left(c_{i j k l} \varepsilon_{k l}(u)\right) v_{i}+\frac{\partial}{\partial x_{i}}\left(c_{j i k l} \varepsilon_{k l}(u)\right) v_{j}\right)+f^{T} v\right) d x \\
&=-\sum_{i} \int_{\Omega}\left(\left(\sum_{j, k, l} \frac{\partial}{\partial x_{j}}\left(c_{i j k l} \varepsilon_{k l}(u)\right)+f_{i}\right) v_{i}\right) d x \\
& \forall v=\left(v_{1}, \ldots, v_{d}\right)^{T} \in\left(C_{0}^{\infty}(\Omega)\right)^{d},
\end{aligned}
$$

which yields (1.1) due to the density $\overline{C_{0}^{\infty}(\Omega)}=H^{1}(\Omega)$.

We proceed analogously to derive the boundary condition (1.2). We use the just derived (1.1), again the symmetry of $a(\cdot, \cdot)$ and $c_{i j k l}$, Green's formula and (2.14) to get

$$
\begin{aligned}
0 & =a(v, u)-b(v) \\
& =\int_{\partial \Omega}\left(\frac{1}{2} \sum_{i, j, k, l}\left(n_{j} c_{i j k l} \varepsilon_{k l}(u) v_{i}+n_{i} c_{j i k l} \varepsilon_{k l}(u) v_{j}\right)-g^{T} v\right) d s \\
& =\sum_{i} \int_{\partial \Omega}\left(\sum_{j, k, l} n_{j} c_{i j k l} \varepsilon_{k l}(u)-g_{i}\right) v_{i} d s \quad \forall v=\left(v_{1}, \ldots, v_{d}\right)^{T} \in V .
\end{aligned}
$$

Denote by $H^{1 / 2}(\partial \Omega)$ the space of traces of all functions from $H^{1}(\Omega)$. Then due to $[7$, p. 87$]$ we have the following density:

$$
L^{2}(\partial \Omega)=\overline{H^{1 / 2}(\partial \Omega)},
$$


where the closure is taken under the $\|\cdot\|_{0, \partial \Omega}$-norm. From here and (2.16) we find that (1.2) holds.

TheOREm 2.5. Let $u \in\left(C^{2}(\bar{\Omega})\right)^{d}$ be the classical solution of problem (1.1)(1.2). Then $u$ is the variational solution of problem (2.3) provided (2.5) holds.

P r o of. Let $v=\left(v_{1}, \ldots, v_{d}\right)^{T} \in V$ be arbitrary and let us multiply (1.1) by $v_{i}$. Then the integration over $\Omega$, summation over $i=1, \ldots, d$, and the use of Green's formula yield (as in (2.15) and (2.16))

$$
\begin{aligned}
0 & =-\sum_{i, j, k, l} \int_{\Omega}\left(\left(\frac{\partial}{\partial x_{j}}\left(c_{i j k l} \varepsilon_{k l}(u)\right)+f_{i}\right) v_{i}\right) d x \\
& =\sum_{i, j, k, l}\left(\int_{\Omega}\left(\frac{1}{2} c_{i j k l} \varepsilon_{k l}(u)\left(\frac{\partial v_{i}}{\partial x_{j}}+\frac{\partial v_{j}}{\partial x_{i}}\right)-f_{i} v_{i}\right) d x-\int_{\partial \Omega} n_{j} c_{i j k l} \varepsilon_{k l}(u) v_{i} d s\right) .
\end{aligned}
$$

Hence, by (1.2), (1.3), (1.11) and (2.2) we have

$$
0=a(v, u)-b(v) \quad \forall v \in V .
$$

Finally, from $(2.1),(2.5)$ and the symmetry of $a(\cdot, \cdot)$ we arrive at

$$
\widetilde{a}(u, v)=a(u, v)=b(v) \quad \forall v \in V .
$$

Remark 2.6. Since $\widetilde{a}(\cdot, \cdot)$ is symmetric, the weak formulation $(2.3)$ of the Neumann problem is obviously equivalent to minimizing the functional $J(v)=$ $\frac{1}{2} \widetilde{a}(v, v)-b(v)$ over $V$ for arbitrary positive $\gamma_{1}, \ldots, \gamma_{D}$. This is the so-called variational formulation. Such an approach leads, by (2.1), to exact penalties methods (see $[5,11])$.

3. Finite element approximation. Throughout this section we assume that $V_{h} \subset V$ is an arbitrary finite element space such that $P \subset V_{h}$. Let the set $S$ from (1.10) be chosen so that $\bar{S}$ is a union of some faces of several elements. A discrete analogue of problem (2.3) consists in finding $u_{h} \in V_{h}$ so that

$$
\widetilde{a}\left(u_{h}, v_{h}\right)=b\left(v_{h}\right) \quad \forall v_{h} \in V_{h} .
$$

Theorem 3.1. There exists a unique solution $u_{h} \in V_{h}$ of problem (3.1). This solution satisfies the conditions

$$
q_{i}\left(u_{h}\right)=0, \quad i=1, \ldots, D,
$$

where the $q_{i}$ are defined by (1.10).

The proof is similar to that of Theorem 2.2.

Theorem 3.2. The discrete solution $u_{h}$ of problem (3.1) is independent of the parameters $\gamma_{i}$ from (2.1).

P r o of. Let $u_{h}^{1}$ and $u_{h}^{2}$ be two solutions of (3.1) corresponding to two different sequences $\left\{\gamma_{i}^{1}\right\}$ and $\left\{\gamma_{i}^{2}\right\}$, respectively. Then, by (3.2),

$$
q_{i}\left(u_{h}^{1}\right)=q_{i}\left(u_{h}^{2}\right)=0, \quad i=1, \ldots, D .
$$


From this and (2.1) we see that

$$
\widetilde{a}\left(u_{h}^{k}, v_{h}\right)=a\left(u_{h}^{k}, v_{h}\right)=b\left(v_{h}\right) \quad \forall v_{h} \in V_{h}, k=1,2,
$$

which yields

$$
a\left(u_{h}^{1}-u_{h}^{2}, v_{h}\right)=0 \quad \forall v_{h} \in V_{h} .
$$

Setting here $v_{h}=u_{h}^{1}-u_{h}^{2}$, we find by (2.6) that

$$
C\left\|\varepsilon\left(u_{h}^{1}-u_{h}^{2}\right)\right\|_{0, \Omega}^{2} \leq a\left(u_{h}^{1}-u_{h}^{2}, u_{h}^{1}-u_{h}^{2}\right)=0,
$$

which implies that $\varepsilon\left(u_{h}^{1}-u_{h}^{2}\right)=0$ almost everywhere in $\Omega$. Hence, by (3.3) and (2.4), we have $u_{h}^{1}-u_{h}^{2}=0$.

Let $m=\operatorname{dim} V_{h}$ and let $\left\{v^{i}\right\}_{i=1}^{m}$ be a finite element basis of $V_{h}$. Set

$$
\widetilde{A}=\left(\widetilde{a}\left(v^{i}, v^{j}\right)\right)_{i, j=1}^{m}, \quad A=\left(a\left(v^{i}, v^{j}\right)\right)_{i, j=1}^{m} .
$$

Notice that the width of the band of the stiffness matrix $\widetilde{A}$ depends upon the choice of the set $S$ occurring in (1.10). We will illustrate this fact in the following example.

EXAmple 3.3 . Let $\Omega=(0,1) \times(0,1)$ and let $N>1$ be a given integer. Divide each side of $\bar{\Omega}$ into $N-1$ equal parts and consider a uniform triangulation of $\bar{\Omega}$ as sketched in Figure 1.

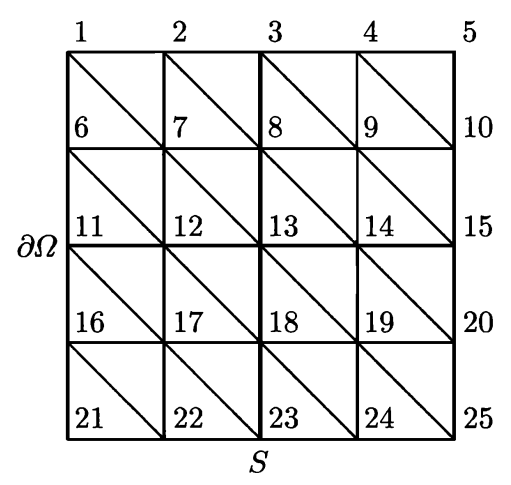

Fig. 1

Assume that the nodes $x^{j}, j=1, \ldots, N^{2}$, are numbered e.g. row-wise and denote by $\varphi^{i}$ the standard Courant piecewise linear basis functions, i.e., $\varphi^{i}\left(x^{j}\right)=\delta_{i j}$ for $i, j=1, \ldots, N^{2}$. Let the space $V_{h}$ be generated by continuous piecewise linear vector fields over each triangulation. The functions

$$
v^{2 i-1}=\left(\varphi^{i}, 0\right)^{T}, \quad v^{2 i}=\left(0, \varphi^{i}\right)^{T}, \quad i=1, \ldots, m\left(=2 N^{2}\right),
$$

can obviously be taken as a basis in $V_{h}$. Then the symmetric matrix $A$ from (3.4) has a band structure. The half bandwidth equals $2 N+2$. We can easily see by (2.1) that the half bandwidth of $\widetilde{A}$ will be the same if we choose $S$ from (1.10) as the lower (or upper) side of $\bar{\Omega}-\mathrm{cf}$. Figure 1 . On the other hand, although 
the choice $S=\partial \Omega$ would yield a sparse matrix $\widetilde{A}$, the band structure would be lost. That is why the set $S$ has to be chosen appropriately. Let us note that the choice of $\gamma_{1}, \ldots, \gamma_{D}$ from (2.1) has no influence upon the structure of $\widetilde{A}$, but has a certain influence on the condition number of $\widetilde{A}$ (see [5]).

Let us further note that the classical setting of the discrete Neumann problem consists in minimizing the quadratic functional

$$
I(y)=y^{T} A y-2 y^{T} \mathbf{b}
$$

subject to the linear constraints

$$
Q y=0,
$$

where $A$ given in (3.4) is singular, $\mathbf{b}=\left(b\left(v^{i}\right)\right)_{i=1}^{m}$ and $Q$ is a $D \times m$ matrix corresponding to a discretization of the constraints $q_{i}(v)=0(i=1, \ldots, D)$ from (1.12). The method of Lagrange's multipliers applied to (3.5)-(3.6) then yields the system (cf. [6, p. 1263])

$$
\left(\begin{array}{cc}
A & Q^{T} \\
Q & 0
\end{array}\right)\left(\begin{array}{l}
y \\
\lambda
\end{array}\right)=\left(\begin{array}{l}
\mathbf{b} \\
0
\end{array}\right)
$$

whose matrix is nonsingular but indefinite. On the contrary, formulation (3.1) leads to the system

$$
\widetilde{A} y=\mathbf{b},
$$

whose matrix is positive definite.

R e m a rk 3.4. The problem of convergence of $\left\|u-u_{h}\right\|_{1, \Omega}$, where $u$ and $u_{h}$ are defined by (2.3) and (3.1), respectively, can be transformed by Céa's well-known lemma (see e.g. [4, p. 41]) to the study of approximation properties of the system $\left\{V_{h}\right\}$ of finite element subspaces in $V$.

Re mark 3.5. According to [2, p. 309 or p. 317], Lemma 2.1 remains valid if the integration domain $S$ in (1.10) is replaced by $\Omega_{i}$ or $S_{i}, i=1, \ldots, D$, where $\Omega_{i} \neq \emptyset$ are subdomains of $\Omega$ with Lipschitz boundaries and $S_{i} \neq \emptyset$ are arbitrary open parts of $\partial \Omega_{i}$. Thus all the previous theorems may be formulated also for this case.

4. Periodic boundary conditions. Assume that $\Omega$ is rectangular, $\Omega=$ $\left(0, r_{1}\right) \times \ldots \times\left(0, r_{d}\right)$, and consider the problem given by (1.1) with the following periodic boundary conditions:

$$
\begin{aligned}
u_{\mid F_{m}} & =u_{\mid F_{m}^{\prime}}, \quad m=1, \ldots, d, \\
\sum_{j, k, l=1}^{d} n_{j} c_{i j k l} \varepsilon_{k l}(u)_{\mid F_{m}} & =\sum_{j, k, l=1}^{d} n_{j} c_{i j k l} \varepsilon_{k l}(u)_{\mid F_{m}^{\prime}}, \quad i, m=1, \ldots, d,
\end{aligned}
$$

where $F_{m}$ and $F_{m}^{\prime}$ are opposite faces of $\Omega$ which are perpendicular to the axis $x_{m}, m=1, \ldots, d$, and $c_{i j k l}$ are from $C^{1}(\bar{\Omega})$ for the time being. The necessity of 
solving such a problem arises in studying materials with periodic structure. For single elliptic equations with periodic boundary conditions see e.g. $[9,10]$. Set

$$
W=\left\{w \in V \mid w_{\mid F_{m}}=w_{\mid F_{m}^{\prime}}, m=1, \ldots, d\right\}
$$

and suppose that some smooth $u$ satisfies (1.1)+(4.1). Multiplying (1.1) by an arbitrary function $w \in W$, integrating this over $\Omega$ and using (1.3), (1.4), the Green formula and (4.1), we come to

$$
a(u, w)=b(w) \quad \forall w \in W,
$$

where $a(\cdot, \cdot)$ is defined in (1.11), $c_{i j k l}$ may belong to $L^{\infty}(\Omega)$ now, and

$$
b(w)=\int_{\Omega} f^{T} w d x, \quad w \in W .
$$

Since the functions $p^{i}, i=1, \ldots, d$, given by (1.9), are constant and belong to $W$, we find by (1.11) and (4.3) that

$$
b\left(p^{i}\right)=0, \quad i=1, \ldots, d .
$$

Note that $a(\cdot, \cdot)$ is not $W$-elliptic, since

$$
a\left(p^{i}, p^{i}\right)=0, \quad i=1, \ldots, d .
$$

It is elliptic only over the space

$$
U=\left\{v \in V \mid q_{i}(v)=0, v_{\mid F_{i}}=v_{\mid F_{i}^{\prime}}, i=1, \ldots, d\right\},
$$

where the $q_{i}$ are defined in (1.10). The space $U$ is, however, unsuitable for finite element approximation, as FE-basis functions would have too complicated shape due to the constraints occurring in $U$. Thus analogously to (2.1), we introduce a modified bilinear form

$$
\stackrel{*}{a}(v, w)=a(v, w)+\sum_{i=1}^{d} \gamma_{i} q_{i}(v) q_{i}(w), \quad v, w \in W,
$$

where $\gamma_{i}>0$ are fixed constants. Consider now a related problem: Find $u \in W$ such that

$$
\stackrel{*}{a}(u, w)=b(w) \quad \forall w \in W .
$$

THEOREM 4.1. There exists a unique solution $u \in W$ of (4.8). This solution satisfies the conditions

$$
q_{i}(u)=0, \quad i=1, \ldots, d .
$$

P r o of. We first prove by contradiction that

$$
\|w\|_{1, \Omega}^{2} \leq C\left(\|\varepsilon(w)\|_{0, \Omega}^{2}+\sum_{i=1}^{d} q_{i}^{2}(w)\right) \quad \forall w \in W .
$$


So, let there exist a sequence $\left\{w_{m}\right\}_{m=1}^{\infty} \subset W$ such that

$$
\left\|w_{m}\right\|_{1, \Omega}^{2}>m\left(\left\|\varepsilon\left(w_{m}\right)\right\|_{0, \Omega}^{2}+\sum_{i=1}^{d} q_{i}^{2}\left(w_{m}\right)\right) .
$$

We may assume, moreover, that the $w_{m}$ are normalized so that

$$
\left\|w_{m}\right\|_{1, \Omega}=1, \quad m=1,2, \ldots
$$

From (4.11) and (4.12) we see that

$$
\left\|\varepsilon\left(w_{m}\right)\right\|_{0, \Omega} \rightarrow 0 \quad \text { as } m \rightarrow \infty,
$$

and for any $i \in\{1, \ldots, d\}$,

$$
q_{i}\left(w_{m}\right) \rightarrow 0 \quad \text { as } m \rightarrow \infty .
$$

Since the imbedding $H^{1}(\Omega) \subset L^{2}(\Omega)$ is compact, there exist $w_{0} \in\left(L^{2}(\Omega)\right)^{d}$ and a subsequence of $\left\{w_{m}\right\}$, still denoted by $\left\{w_{m}\right\}$, such that

$$
\left\|w_{m}-w_{0}\right\|_{0, \Omega} \rightarrow 0 \quad \text { as } m \rightarrow \infty .
$$

Using now the coercivity of strains, i.e.

$$
C\|v\|_{1, \Omega}^{2} \leq\|\varepsilon(v)\|_{0, \Omega}^{2}+\|v\|_{0, \Omega}^{2} \quad \forall v \in V
$$

(see e.g. [8, p. 79]), we get for any $m, r \in\{1,2, \ldots\}$

$$
\begin{aligned}
& C\left\|w_{m}-w_{r}\right\|_{1, \Omega}^{2} \\
& \quad \leq\left\|\varepsilon\left(w_{m}\right)\right\|_{0, \Omega}^{2}+2\left\|\varepsilon\left(w_{m}\right)\right\|_{0, \Omega}\left\|\varepsilon\left(w_{r}\right)\right\|_{0, \Omega}+\left\|\varepsilon\left(w_{r}\right)\right\|_{0, \Omega}^{2}+\left\|w_{m}-w_{r}\right\|_{0, \Omega}^{2} .
\end{aligned}
$$

This estimate, (4.13) and (4.15) imply that $\left\{w_{m}\right\}$ is a Cauchy sequence in $V$. Applying (4.15) once again, we find that $w_{0} \in V$ and that

$$
\left\|w_{m}-w_{0}\right\|_{1, \Omega} \rightarrow 0 \quad \text { as } m \rightarrow \infty .
$$

Since all $w_{m}$ belong to $W$, we further deduce by the trace theorem that also $w_{0} \in W$. Moreover, (4.13) and (4.16) yield

$$
\varepsilon\left(w_{0}\right)=0 \text {. }
$$

From this and (1.9) we observe that $w_{0}$ must be constant on $\Omega$ as $w_{0} \in W$ is periodic. Finally, from definition (1.10) we get

$$
w_{0}=0,
$$

since by $(2.8),(4.16)$ and $(4.14)$ we have $q_{i}\left(w_{0}\right)=0$ for $i=1, \ldots, d$. However, (4.17) contradicts (4.12) and (4.16), i.e., (4.10) holds.

Now from (4.7), (1.5) and (4.10), we observe that $\stackrel{*}{*}(\cdot, \cdot)$ is $W$-elliptic, i.e.

$$
\stackrel{*}{a}(w, w)=a(w, w)+\sum_{i=1}^{d} \gamma_{i} q_{i}^{2}(w) \geq C_{1}\|\varepsilon(w)\|_{0, \Omega}^{2}+C_{2} \sum_{i=1}^{d} q_{i}^{2}(w) \geq C_{3}\|w\|_{1, \Omega}^{2}
$$

for all $w \in W$. Since, moreover, the forms (4.4) and (4.7) are continuous, the existence of a unique solution $u \in W$ follows from the Lax-Milgram lemma. 
Further we prove (4.9). By (2.7), (4.7), (4.8) and (4.5),

$$
\sum_{i=1}^{d} \gamma_{i} q_{i}(u) q_{i}\left(p^{j}\right)=a\left(u, p^{j}\right)+\sum_{i=1}^{d} \gamma_{i} q_{i}(u) q_{i}\left(p^{j}\right)=\stackrel{*}{a}\left(u, p^{j}\right)=b\left(p^{j}\right)=0
$$

for any $j=1, \ldots, d$. Therefore, from (2.10) and (2.11) we come to (4.9).

R e mark 4.2. Theorems 2.4, 2.5 and Remark 2.6 can be stated analogously also for periodic boundary conditions. If $W_{h} \subset W$ is an arbitrary finite element space which contains constant functions, then a discrete analogue of (4.8) consists in finding $u_{h} \in W_{h}$ such that

$$
\stackrel{*}{a}\left(u_{h}, w_{h}\right)=b\left(w_{h}\right) \quad \forall w_{h} \in W_{h} .
$$

Theorems 3.1, 3.2 and Remarks 3.4 and 3.5 can be again easily modified to the space $W_{h}$. Therefore, the discrete solution $u_{h}$ is independent of $\gamma_{1}, \ldots, \gamma_{d}$, but the stiffness matrix associated with (4.18) and some basis $\left\{w^{i}\right\} \subset W_{h}$ essentially depends upon $\gamma_{1}, \ldots, \gamma_{d}$. This can be seen from the following numerical example.

EXAMPLE 4.3. Let $d=3$ and suppose that the elastic body $\Omega=(0,1) \times(0,1) \times$ $(0,1)$ consists of a homogeneous and isotropic material with Lamé's constants $\mu=c_{1212}=10^{11}\left[\mathrm{Nm}^{-2}\right]$ and $\lambda=c_{1122}=10^{11}\left[\mathrm{Nm}^{-2}\right]$. We choose the right-hand side of (1.1) so that

$$
u_{1}\left(x_{1}, x_{2}, x_{3}\right)=\frac{1}{100} \sin \left(2 \pi x_{1}\right), \quad u_{2} \equiv u_{3} \equiv 0
$$

is the true solution of the problem $(1.1)+(4.1)$. Let each edge of $\bar{\Omega}$ be divided into $N$ equal parts and let $W_{h}$ be the corresponding vector finite element space (from (4.18)) generated by the standard trilinear elements, i.e., $\operatorname{dim} W_{h}=3 N^{3}$. Let us number the basis functions $w^{i}$ lexicographically. In order to define the bilinear form (4.7), we further set $S=\{0\} \times(0,1) \times(0,1)$ and $\gamma_{1}=\gamma_{2}=\gamma_{3}=\gamma$, where $\gamma$ is a positive parameter. Due to (4.2), the stiffness matrix $\left(\stackrel{*}{a}\left(w^{i}, w^{j}\right)\right)$ associated with (4.18) will not be a band matrix (as in Example 3.3), but will remain sparse. The system of simultaneous equations has been solved iteratively by the SOR method with $\omega=1.4$ and zero initial guess in all cases. In the first row of Table 1 we see the values of the expression

$$
\frac{\stackrel{*}{a}\left(w^{1}, w^{1}\right)-a\left(w^{1}, w^{1}\right)}{a\left(w^{1}, w^{1}\right)} \cdot 100 \%
$$

for various values of the parameter $\gamma$. The iteration process has been stopped

Table 1

\begin{tabular}{|c|r|c|c|c|c|}
\hline & $3 \%$ & $7 \%$ & $15 \%$ & $30 \%$ & $300 \%$ \\
\hline$N=3$ & 80 & 29 & 25 & 24 & 23 \\
$N=4$ & 110 & 51 & 28 & 26 & 25 \\
$N=5$ & 138 & 72 & 36 & 32 & 26 \\
\hline
\end{tabular}


when the Euclidean norm of two subsequent iterations was less than $10^{-6}$. The corresponding numbers of iterations are given in Table 1.

\section{References}

[1] I. Babuška Uncertainties in engineering design: mathematical theory and numerical experience, in: The Optimal Shape, J. Bennett and M. M. Botkin (eds.), Plenum Press, 1986; also in: Technical Note BN-1044, Univ. of Maryland, 1985, 1-35.

[2] I. Hlaváček and J. Nečas, On inequalities of Korn's type, I, Boundary-value problems for elliptic systems of partial differential equations, II, Applications to linear elasticity, Arch. Rational Mech. Anal. 36 (1970), 305-311, 312-334.

[3] M. Kř́žžek, Conforming equilibrium finite element methods for some elliptic plane problems, RAIRO Numer. Anal. 17 (1983), 35-65.

[4] M. Kř́žžek and P. Neittaanmäki, Finite Element Approximation of Variational Problems and Applications, Longman, Harlow 1990.

[5] M. Kř́žžek, P. Neittaanmäki and M. Vondrák, A nontraditional approach for solving the Neumann problem by the finite element method, Mat. Apl. Comput. 11 (1992), 31-40.

[6] D. F. Luenberger, Hyperbolic pairs in the method of conjugate gradients, SIAM J. Appl. Math. 17 (1969), 1263-1267.

[7] J. Nečas, Les méthodes directes en théorie des équations elliptiques, Academia, Prague 1967.

[8] J. Nečas and I. Hlaváček, Mathematical Theory of Elastic and Elasto-plastic Bodies: An Introduction, Elsevier, Amsterdam 1981.

[9] J. Taufer, Lösung der Randwertprobleme für Systeme von linearen Differentialgleichungen, Academia, Prague 1973.

[10] R. Temam, Infinite-dimensional Dynamical Systems in Mechanics and Physics, Springer, Berlin 1988 .

[11] D. E. Ward, Exact penalties and sufficient conditions for optimality in nonsmooth optimization, J. Optim. Theory Appl. 57 (1988), 485-499. 